\title{
Civilisations
}

Revue internationale d'anthropologie et de sciences

humaines

$62 \mid 2013$

Identité, culture et intimité

\section{Une petite production de soi dans l'entre-soi}

Actualisations quotidiennes d'une mémoire post-migratoire

\section{Stéphanie Tabois}

\section{(2) OpenEdition}

\section{Journals}

Édition électronique

URL : http://journals.openedition.org/civilisations/3343

DOI : 10.4000/civilisations.3343

ISSN : 2032-0442

Éditeur

Institut de sociologie de l'Université Libre de Bruxelles

\section{Édition imprimée}

Date de publication : 31 décembre 2013

Pagination : 149-164

ISBN : 2-87263-042-2

ISSN : 0009-8140

\section{Référence électronique}

Stéphanie Tabois, « Une petite production de soi dans l'entre-soi », Civilisations [En ligne], 62 | 2013, mis en ligne le 31 décembre 2016, consulté le 02 mai 2019. URL : http://journals.openedition.org/ civilisations/3343; DOI : 10.4000/civilisations.3343 


\title{
Une petite production de soi dans l'entre-soi Actualisations quotidiennes d'une mémoire post-migratoire
}

\author{
Stéphanie TABOIS
}

Résumé : Délibérément inscrit dans une perspective micrologique, les analyses présentées dans cet article interrogent l'actualisation de la mémoire individuelle sur son versant situationnel, à partir d'une enquête de terrain réalisée auprès de la population pied-noire dans le milieu des Pieds-Noirs. L'objectif de ce texte consiste à mettre au jour une partie du travail permanent d'appropriation et de reformulation de la mémoire ; un processus qui prend forme dans le détail des interactions ordinaires de la vie quotidienne. L'examen des données recueillies dans différents contextes micro-sociaux privés montre comment des éléments, sinon vrais, du moins plausibles, se maintiennent, sont réévalués ou introduits dans la mémoire individuelle. L'actualisation des cadres et contenus mnésiques, à la fois reconduits et amendés au gré d'échanges banals, obéit donc aux dynamiques propres aux interactions ordinaires, qui à ce titre, apparaissent comme des sources relativement autonomes de la " petite production mnésique».

Mots-clefs : mémoire, interactions banales, quotidien, cadres sociaux, sociabilité, stéréotypes.

\begin{abstract}
Based on a fieldwork among the French "Pieds-Noirs," this article analyses through a "micro" perspective the enactment of individual memory in concrete situations. Its aim is to decipher elements of the everyday processes of appropriation and reformulation of memory, as it takes place in ordinary interactions. The analysis of these interactions observed in very detailed settings shows how elements that are at least believable (if not "true") are produced, maintained, reevaluated and cast in one's memory. The actualization of those frames and contents of memory seems eventually to follow the modalities of banal interactions, which can hence be considered as relatively autonomous source of the "small production of memory".
\end{abstract}

Keywords: memory, banal interactions, everyday life, social frames, sociability, stereotypes. 
"Comptons, dans une journée, le nombre de souvenirs que nous avons évoqués à l'occasion de nos rapports directs et indirects avec d'autres hommes. Nous verrons que, le plus souvent, nous ne faisons appel à notre mémoire que pour répondre à des questions que les autres nous posent, ou que nous supposons qu'ils pourraient nous poser, et que d'ailleurs, pour y répondre, nous nous plaçons à leur point de vue, et nous nous envisageons comme faisant partie du même groupe ou des mêmes groupes qu'eux. »

Les cadres sociaux de la mémoire, Maurice Halbwachs.

Le rapport que les Pieds-Noirs entretiennent avec l'Algérie est fondamentalement tributaire d'une mémoire de la guerre et de l'exil. Par la signature des Accords d'Evian en 1962, la France a libéré cette colonie d'une tutelle qu'elle lui imposait depuis plus de cent trente ans. La plupart des Européens vivant jusqu'alors dans ce département français a dû quitter précipitamment son sol natal. Ils abandonnaient ainsi un territoire sur lequel nombre d'entre eux avaient fait souche depuis plusieurs générations ${ }^{1}$.

Bien que la grande majorité des Pieds-Noirs encore en vie ait désormais vécu plus longtemps en Métropole qu'en Algérie, ceux-ci présentent aujourd'hui encore cette particularité de se représenter comme exilés dans leur propre pays. La question de leur identité fait dès lors problème ; elle se pose avec d'autant plus de force qu'elle est vécue dans une distorsion entre l'attachement à un monde de référence - un paradis perdu - et un rapport ambigu à la Métropole.

Aujourd'hui, hors de la communauté, la mémoire des Pieds-Noirs est bien souvent considérée comme illégitime ; un caractère indissociablement lié à la situation de domination coloniale passée, à laquelle les membres de ce groupe ont, bon gré mal gré, participé. Quelles qu'aient pu être leurs positions personnelles à l'égard du statut de l'Algérie, « le fait même d'avoir accepté de s'[y] installer en tant que Français (de ne pas en être parti s'il y est né) condamne l'individu à un statut d'oppresseur. Cela imprègne aujourd'hui encore l'imaginaire de tout rapatrié d'Algérie » (Buono 2004 : 35). Dans les regards qu'ils portent sur eux-mêmes, les Pieds-Noirs oscillent ainsi entre deux pôles : une puissante identité de « Méditerranéen », mais qui, dans le même temps, ne parvient pas à trouver ses étais, c'est-à-dire des traits identitaires acceptables et valorisés par tous. Comment, en effet, tirer un parti satisfaisant d'un accent trop souvent raillé, de manières d'être ou de vivre ne présentant pas de formes suffisamment spécifiques, tandis encore que l'exotisme dont ils pourraient se prévaloir souffre de son caractère suranné et, surtout, se trouve immédiatement parasité par les images du passé colonial de la France?

Dès les premières rencontres avec des Pieds-Noirs, nous avons pu constater combien cet aspect illégitime représente un enjeu du point de vue collectif, combien il semble complexe pour les individus de se situer entre sentiments et souvenirs individuels, mémoire revendiquée par les associations, mémoire nationale officielle, etc. (Herzfeld 1997). Ces tensions se retrouvent alors de fait dans les modalités d'appropriation

1 Les historiens estiment généralement que l'exil d'Algérie a concerné près d'un million de personnes (cf. par exemple Stora 1993). 
individuelle des trajectoires biographiques qui donnent à voir les articulations entre « poids » et « choix » du passé (Lavabre 1991).

La rupture avec les conditions sociales d'existence en Algérie a disloqué les familles mais elle a également mis à mal les réseaux sociaux de chacun. Les amitiés ont rarement survécu au déracinement, ce dernier ayant réduit à néant le capital social de la plupart des enquêtés. Les Pieds-Noirs ont ainsi dû recomposer leur cercle amical dans le contexte métropolitain, et ils y sont parvenus avec plus ou moins de bonheur.

Dans les premiers temps de leur installation, ils ne se sont guère souciés de recréer des liens amicaux, cherchant avant tout à s'intégrer à leurs groupes d'accueil. Les retrouvailles entre amis étaient rarissimes, souvent laissées au hasard. Les associations d'anciens Français d'Algérie ont alors joué un rôle essentiel dans le tissage de nouvelles relations. Ces temps et ces lieux voués aux retrouvailles ont ainsi été institués au fil des années par différents groupes associatifs ; ils demeurent néanmoins ponctuels et ne représentent jamais que des pis-aller. Les réunions d'anciens issus de tel village, de tel quartier, lycée, club sportif, etc. ont malgré tout largement contribué à créer des liens avec d'autres compatriotes exilés.

En dehors des associations de migrants, les pratiques de loisirs ont donné l'occasion à certains de rencontrer des "Métropolitains $»^{2}$. Mais, les Pieds-Noirs interrogés affirment apprécier davantage de pouvoir échanger avec leurs semblables. Ils s'attribuent entre eux certaines qualités et considèrent qu'ils échangent sur un mode de relation (perçu comme " méditerranéen ») qui leur est propre. Ainsi, dès que des opportunités se présentent à eux, les Pieds-Noirs prisent davantage les amitiés nouées dans l'entre-soi ${ }^{3}$. Après C. Bidart, nous avons pu constater ici la force de l'homogamie sociale dans cette " nette tendance à, de façon générale, " préférer le même que soi » " (2006 : 216).

Le propos de cet article tire parti des analyses d'un corpus d'entretiens (un peu plus de 70) réalisés avec des Pieds-Noirs, associé à un ensemble d'observations menées dans les espaces du groupe - aussi bien publics (i.e. commémorations) que privés (i.e. espaces domestiques). Exposées dans le cadre d'une thèse de doctorat, elles visaient à saisir les conditions pratiques de constitution et d'émergence quotidiennes de la mémoire des Pieds-Noirs. La recherche tenait, en particulier, compte de la présence des objets rappelant l'Algérie dans les environnements familiers (notamment domestiques).

Il s'agit ici de conserver cette perspective micrologique sur la formation de la mémoire individuelle, en choisissant d'explorer plus précisément le versant situationnel de cette dernière. L'objectif de ce texte consiste à mettre au jour les manifestations concrètes de formes collectives de catégorisation, ainsi que les modes d'expression privés du sentiment d'un passé partagé. Nous proposons, à cette fin, une étude détaillée de deux contextes d'observation : une réunion festive associative et une rencontre amicale entre trois couples. Ces données, recueillies par observation participante, nous ont paru présenter les traits les plus significatifs des formes d'échanges amicaux que

2 L'existence et le maintien de l'usage de ce terme spécifique signifiant bien que les Pieds-Noirs continuent à percevoir les « Métropolitains » comme des « Autres ».

3 Ce qui ne revient pas à dire qu'ils ne possèdent pas d'amis parmi les Métropolitains. Notre objectif consistant ici à comprendre la manière dont les relations amicales peuvent avoir des effets sur les mémoires, nous examinons plus spécifiquement les conséquences des affinités entre Pieds-Noirs. 
nous avons pu identifier tout au long de l'enquête. Elles nous permettront d'examiner la manière dont la mémoire individuelle s'exprime, se maintient et se constitue, par le biais de stéréotypes, dans le détail des interactions ordinaires de la vie quotidienne ${ }^{4}$.

\section{Une première scène de la conversation banale : l'entre-soi associatif}

L'observation présentée ici porte sur des festivités associatives. La particularité de ce type de réunion à vocation communautaire tient au fait que chacun des participants sait (ou suppose) que ses interlocuteurs sont présents parce qu'ils partagent une histoire et une trajectoire similaires à la sienne.

Nous présenterons ici certaines séquences d'interaction, afin d'analyser la nature, le type et le contenu des échanges à tonalité mnésique qui prennent forme dans des espaces semi-privés.

Conviée par une informatrice à un déjeuner, organisé deux à trois fois par an par une association pied-noir afin de réunir un maximum d'adhérents, nous nous présentons à l'heure dite, comme cela avait été demandé avec quelques kémias et des couverts ${ }^{5}$. Bien que le repas ait été confié à un traiteur, il est, en effet, suggéré à chaque participant de contribuer à l'apéritif (l'association fournit, en revanche, les boissons, notamment l'anisette, boisson identitaire pour les Pieds-Noirs).

Nous retrouvons alors notre informatrice qui s'affaire aux côtés d'autres femmes dans la salle de réunion de l'association. Elles y transportent les rouleaux de nappes en papier et y déplacent tables et chaises. Une quinzaine de personnes sont déjà présentes et, bien qu'il ne nous connaisse pas, un homme nous sollicite afin d'installer le buffet de kémias. Nous nous présentons mais Clément $^{6}$, notre interlocuteur, semble faire bien peu de cas de nos explications. Il retient uniquement le fait que «Stéphanie est l'amie de Madame F. $»^{7}$. Nous intégrons ainsi un petit groupe composé de trois hommes et d'une femme (tous âgés d'environ soixante-cinq ans). Les hommes disposent les bouteilles de jus de fruit, d'eau et d'anisette (seul alcool disponible pour l'apéritif). Avec Béatrice, nous nous chargeons de présenter les kémias dans des assiettes jetables.

4 Cet article porte sur la description et l'analyse de phénomènes micrologiques. La volonté de conserver à ce texte son ancrage empirique et d'approfondir l'étude de chaque situation présentée, commande de ne laisser apparaître les soubassements théoriques qu'en filigrane. Précisions toutefois que nous situons notre approche à la croisée des travaux de M. Halbwachs (1994 (1925) ; 1997 (1950)), de R. Bastide (1970) et de la sociologie phénoménologique d'A. Schütz (1994 (1971)) et de P. Berger et T. Luckmann (1996 (1966)).

5 Le terme « kémias » désigne des aliments salés destinés à être grignotés au cours de l'apéritif (il s'agit de l'équivalent des tapas espagnoles).

6 Tous les noms et prénoms des personnes ont été modifiés.

7 A. Piette écrit que « c'est directement à partir de cette interprétation pertinente localement (c'est-à-dire l'assignation à telle ou telle place) que le chercheur est aidé pour repérer les "classifications indigènes", l'espace des positions et des relations" » (1996: 70). Cette place assignée a minima nous convenait parfaitement. Elle signifiait que, pour Clément au moins, si nous étions là et que nous étions disponible pour l'action, c'est que notre présence était légitime. 
Béatrice: Ca c'est sûr, c'est Madame Brasco ${ }^{8}$ qui a préparé tout ça [elle désigne du doigt plusieurs boîtes en plastique contenant des poissons marinés, de la tortilla, des poivrons à l'huile et un cake salé].

Homme: Elle est bonne cuisinière, il a de la chance Roger [l'époux de Madame Brasco ], c'est pas toi qui ferais pareil, hein!

Béatrice: T'es gonflé!

Nous : C'est plutôt espagnol, tout ça, non?

Béatrice : Oui, Madame Brasco elle est espagnole, elle est spécialiste. Elle est d'Oran.

Homme 2: Qu'est-ce tu dis qu'elle est d'Oran, elle est de Bône !! [rires] C'est son mari qu'est d'Oran.

Béatrice : Elle a quand même vécu à Oran, non ... ?

Homme 2: Oouui [feignant l'exaspération], mais elle, elle est de Bône!

Dans un tel regroupement entre Pieds-Noirs, tous les participants ne se connaissent pas intimement. Si Béatrice (qui est née à Boufarik, dans l'Algérois) était originaire de la même région que Madame Brasco, il ne fait aucun doute qu'elle en saurait davantage sur la biographie de cette dernière, et notamment sur son lieu précis de résidence algérienne. En effet, les membres des associations se regroupent très majoritairement en fonction de leur implantation géographique en Algérie.

Nous remarquons ici qu'afin de catégoriser les personnes avec lesquelles aucun lien nourri n'existe, Béatrice a tendance à orienter les quelques connaissances à sa disposition à la manière dont elle avait l'habitude de lire l'espace des positions en Algérie. Agée de moins de cinquante ans, elle est probablement trop jeune pour avoir expérimenté, en Algérie, ces opérations classificatoires dont elle a cependant intériorisé les principes, au cours des différentes étapes de sa socialisation. Ainsi, pour cette femme, il y a toutes les chances pour qu'une personne cuisinant à l'espagnole ait vécu dans l'Oranie. Béatrice a vraisemblablement recueilli auparavant une information dont elle n'a pas éprouvé le besoin de vérifier le bien-fondé : Madame Brasco aurait vécu à Oran, une donnée corroborée par son style culinaire.

Une telle catégorisation implicite offre un mode confortable de saisie de la réalité quotidienne, dans le sens où elle entretient chez le sujet l'impression de la permanence d'un monde. Ce qui semblait fonctionner cognitivement en Algérie doit demeurer efficient lorsqu'un groupe de Pieds-Noirs est réuni. Plus le sentiment de stabilité trouve à se maintenir, plus il est possible de continuer à croire dans le caractère opératoire des catégories acquises dans l'Algérie coloniale.

Les rencontres entre Pieds-Noirs fonctionnent alors - entre autres choses - comme des occasions de consolider une image typique, simplifiée, du passé. A l'occasion de l'échange que nous avons rapporté, l'image de Madame Brasco telle qu'elle s'était esquissée dans l'esprit de Béatrice se voit amendée et précisée. Ces quelques mots permettent à Béatrice d'en apprendre davantage sur une femme qu'elle rencontre parfois, de revenir sur une approximation et donc, dans une certaine mesure de rendre Madame

8 Remarquons que, pour la plupart d'entre eux, les membres de cette association n'ont pas pour habitude de s'appeler par leur prénom. Cela fournit un indice de la forme de relation qui y a majoritairement cours : les participants se connaissent mais ne sont pas des amis intimes, loin s'en faut. 
Brasco plus familière. Cependant, la relation causale qu'actualise la rationalisation de Béatrice ne se voit pas remise en cause en elle-même : l'ensemble des interactants présents considère ainsi que le fait pour une femme de préparer des recettes espagnoles constitue un indice vraisemblable de son origine oranaise.

Le déjeuner étant prêt, nous sommes invités à passer à table ; nous nous installons (par hasard) près de Béatrice et en face d'un homme nommé Cyprien. Ce dernier, de nature enjouée, anime le repas de ses voisins de table en racontant une foule d'anecdotes le concernant, mêlant certaines aventures algériennes passées à des péripéties plus actuelles. Ses récits sont entrecoupés de conversations sur la vie quotidienne actuelle : l'un se réjouit des économies de chauffage réalisées après l'installation d'un insert, tandis qu'un autre déplore les nuisances sonores causées dans son quartier par la réalisation de travaux de voierie, etc. Notre voisin plaisante sur le fait que nous n'ayons probablement pas compris ce que Béatrice venait de désigner comme un « chiffon de par terre $\|^{9}$. S'ensuivent alors des échanges joyeux sur certains termes considérés par les interlocuteurs comme typiquement pieds-noirs ou sur quelques expressions pataouètes $^{10}$. Jadis stigmatisé, l'accent et les expressions pataouètes se voient aujourd'hui valorisées, en particulier lors de ce type de rassemblement associatif. Dans cet entre-soi, les expressions exotiques et hautes en couleur fusent; les membres de la communauté disent de la sorte leur identité et leur commune appartenance par le biais d'un vocabulaire singularisant qui contribue à délimiter les frontières du groupe. Ainsi, la spécificité de notre position nous a donné accès à de précieuses informations sur les effets de la présence d'un « tiers » sur le cadre des échanges : notre ignorance de ce qui était intimement partagé par les autres convives les incitait à produire des explicitations concernant la vie algérienne. Des explications qui, habituellement, demeuraient efficacement dans l'implicite, renforçant ainsi très discrètement le sentiment du partage d'un monde commun.

Après le déjeuner, le café est servi au buffet. Grâce aux explications de Béatrice et de Cyprien, nous identifions plus aisément une quinzaine de personnes, dont nous connaissons tantôt la profession, tantôt l'origine géographique, le lieu de résidence en Algérie, parfois les loisirs (untel est un redoutable joueur de ping-pong), la situation familiale (untel vient de perdre sa femme), etc.

Nous essayons alors de nous introduire dans quelques-uns des groupes (de deux à cinq personnes) qui se recomposent à proximité des tables, espérant à nouveau pouvoir participer aux échanges. Il y est surtout question de la vie quotidienne actuelle. Les membres de l'un des groupes, constitué de trois hommes et d'une femme, discutent de politique, l'un d'entre eux défendant avec ferveur la " pertinence des interventions » de Marine le Pen; les autres ne démentent pas mais ne partagent visiblement pas son

9 Chez les Pieds-Noirs, cette expression désigne une serpillière.

10 Le pataouète est un sabir fait d'emprunts aux langues du pourtour méditerranéen. Il tend à devenir l'un des symboles d'une « culture pied-noire » désormais farouchement défendue. Sa forme donnant aisément prise à l'exubérance, il rencontre certaines des représentations identitaires des Pieds-Noirs qui revendiquent généralement un naturel entier et généreux. 
enthousiasme ${ }^{11}$. L'un d'entre eux affirme cependant, sur le ton de la révélation : « il y a qu'eux [l'extrême droite] qui nous ont toujours soutenus »; tous acquiescent malgré leurs vraisemblables dissensions (il nous semble avoir compris que les autres membres du groupe se cantonnent plutôt à des positions de droite plus modérées). Cela incite l'un des hommes à parler des conditions de son arrivée en Métropole et du souvenir de désœuvrement de sa mère qu'il y associe. L'échange se poursuit et, emportée par sa dynamique, nous ne remarquons pas combien les propos des uns par rapport à ceux des autres sont décousus. Ce n'est qu'au cours de la prise de notes relative à cette observation en particulier que nous comprenons qu'en réalité, chacun a tenu à raconter sa version du départ, aucun semble-t-il n'écoutant véritablement ce que l'autre pouvait avoir à exprimer. En effet, c'est surtout le cadre processuel du récit et le cheminement suivi qui fonde ce type d'échange. Par exemple :

Homme 1: Il explique qu'il est arrivé à Marseille et rapporte son effroi en voyant la foule, les files d'attente, etc.

Homme 2: Apparemment indifférent au souvenir de malaise qui vient d'être évoqué, il cherche à son tour à faire valoir le fait que débarquer à Port-Vendres était pire encore, les habitants ayant insulté les Français venant d'Algérie.

Femme : $\quad$ Elle juge quant à elle que la vie qu'elle a menée à leur arrivée avec sa famille, pendant plusieurs mois dans un studio, a pesé lourdement dans la séparation de ses parents. Elle est interrompue par un autre homme qui la hèle ; elle quitte le groupe pour le rejoindre.

Homme 2: Il revient sur les insultes qu'il a reçues lorsqu'il se déplaçait en voiture, celle-ci étant encore immatriculée en Algérie.

Pour l'observateur extérieur, chacun des interactants tient à raconter son histoire, sans plus d'égards pour le récit de ces interlocuteurs. Néanmoins, l'apparent « dialogue de sourds » ne donne jamais à voir qu'une forme du « partage », traduisant a minima mais efficacement - « le sentiment subjectif qu'ont les individus membres d'un groupe de partager la même mémoire » (Candau 1999 : 173) ; la présence d'amis permet, en effet, de « se dire » à des personnes que l'on croit réceptives et susceptibles de comprendre sa propre histoire. Selon ce que nous avons pu saisir en situation, ces quatre personnes se connaissent assez peu; le faible degré d'intimité favorise donc la tenue d'échanges qui contribuent à construire une identité biographique sur des formes collectives de catégorisation (concernant ici l'exil, l'arrivée en Métropole et l'installation). Les rencontres entre exilés permettent d'échanger sur son passé, de trouver des points d'appui biographiques communs, de les actualiser, de les mettre à l'épreuve, voire de les construire dans l'interaction.

Au cours de manifestations semblables à celle que nous venons de décrire, nous avons observé que les participants se regroupent majoritairement en fonction de

11 Nous ne sous-entendons pas que, sur le plan politique, les Pieds-Noirs se positionneraient systématiquement à l'extrême-droite. Il s'agit là d'une vision stéréotypée dont E. Comtat (2009) a montré qu'elle ne reflétait que très imparfaitement la réalité de leurs adhésions politiques, mettant à mal l'existence d'un " vote pied-noir ». Les Français d'Algérie, avant 1962 votaient d'ailleurs majoritairement à gauche ; une fois en Métropole, ils se sont cependant massivement tournés vers les partis de droite, les autres condamnant leur vie en Algérie et le colonialisme. 
leur origine géographique algérienne. Les Pieds-Noirs apprécient par-dessus tout, en effet, de pouvoir évoquer les lieux dans lesquels ils ont vécu. Ils éprouvent un vif plaisir à répéter le nom des villes, des villages, des quartiers, de leurs rues, à discuter de l'emplacement précis des commerces, etc. Ils attachent ainsi une importance considérable à la spatialisation de leurs souvenirs ${ }^{12}$.

Ensuite, à l'intérieur de chaque groupe, caractérisé par une origine géographique dominante, les amis se retrouvent selon les points communs et les affinités individuelles. Par exemple, autour d'une pratique de loisir : Mme F., notre personne-ressource, discutait avec deux femmes avec lesquelles elle pratique la marche depuis quelques années; il peut aussi s'agir d'une proximité des conditions de travail passées, soit que celles-ci aient exigé des qualifications identiques, soit que les activités professionnelles aient conduit à fréquenter des connaissances communes.

Ces éléments d'observations invitent ainsi à identifier un double niveau d'homophilie. D'abord, les Pieds-Noirs apprécient de se retrouver entre eux ; ensuite, la composition de leur entourage social obéit à des logiques déjà mises au jour par les chercheurs travaillant sur la sociabilité à dominante élective (origine géographique, génération, profession, etc. $)^{13}$. Néanmoins, d'un point de vue mnésique, ces réflexions permettent de saisir que cette sociabilité joue un rôle dans la mise en forme des souvenirs. Les réunions associatives de ce type fournissent, en effet, des occasions d'actualiser certains éléments mnésiques, souvent personnels mais non intimes. Dans leurs aspects les plus narratifs, ils se voient alors encadrés par les modèles de présentation de soi ayant cours d'une part au sein des associations pieds-noirs, et, d'autre part, dans les milieux socioéconomiques propres aux interactants.

La mémoire des membres des associations se nourrit du contenu mnésique qui s'énonce dans ces rassemblements par le truchement des discours qui y sont tenus par leurs représentants, par les interactions plus banales entre membres, par les livres qui circulent ${ }^{14}$, etc. Mais les mémoires relatives à l'Algérie française sont complexes et multiples. Les spécialistes de l'histoire coloniale pointent en effet la difficulté de les harmoniser. La pluralité des groupes concernés par ce passé, comme les enjeux politiques qui découlent de la volonté d'imposer une mémoire " officielle », une «Vérité », interdisent aujourd'hui encore d'envisager sereinement ce pan de l'histoire de la France et de l'Algérie. Et l'on peut donc affirmer, comme le fait E. Savarèse, à propos de ces multiples appartenance que « personne ne possède les mêmes souvenirs, et, surtout, [que] nul n'a oublié les mêmes épisodes » [2002 : 13]. Chaque sous-groupe, rangé derrière ce que M. Pollak nomme ses « entrepreneurs de mémoire », s’emploie ainsi à promouvoir sa propre version de l'histoire.

La mémoire qui s'exprime alors dans l'entre-soi associatif relève souvent du domaine de la nostalgie sans que, pour autant, il soit systématiquement question de souvenirs référant à des instants de bonheur passés. La proximité relative des Pieds-

12 Cela explique par exemple, qu'à l'occasion du pèlerinage de Santa Cruz à Nîmes, qui réunit tous les ans de très nombreux Pieds-Noirs, les recherches et les retrouvailles s'effectuent avant tout en fonction de la ville ou du quartier d'origine, davantage que selon les fréquentations passées d'écoles, de lycées, de lieux de travail ou de loisirs.

13 Par exemple, $c f$. Bidart (2006).

14 Sur les rapports entre livres, mémoire et identité chez les Pieds-Noirs, $c f$. S. Tabois (2005). 
Noirs présents suffit à fournir l'occasion à des échanges chaleureux, mais, au final, assez peu impliquants. Concernant l'expression de l'identité pied-noir, il semble bien que ce qui se joue dans la co-présence importe davantage que les paroles prononcées. En l'espèce, le sentiment de l'intimité se fonde, dans ces relations de proximité ordinaire, essentiellement sur les sensations de familiarité et d'aisance inhérentes au partage de connaissances banales partagées. Nous inspirant de la tautologie énoncée par C. Javeau (1996 : 255), on pourrait traduire ainsi le principe communicationnel réglant les échanges auxquels nous avons assisté : « je parle de mon histoire de PiedNoir, donc, je suis Pied-Noir, donc puisque tu comprends mon histoire pied-noir tu l'es aussi, puisque je te parle de cette histoire $»$.

Retrouver des amis au sein d'un regroupement associatif permet donc de renouveler un discours sur soi et, ce faisant, de réaffirmer (y compris à soi-même en puisant dans des ressources identitaires) la dimension pied-noir de la définition de soi. En matière de maintien de la mémoire individuelle, ces rencontres sont d'autant plus efficaces qu'elles confirment le sentiment d'appartenance à une communauté affective. En outre, l'homophilie constatée joue vraisemblablement pour beaucoup dans l'absence globale de dissonance mnésique.

\section{Une deuxième scène de la conversation banale : une réunion amicale}

La seconde observation que nous nous proposons d'analyser se déroule dans un contexte domestique. Elle donne à voir une plus grande proximité affective entre les individus présents. Les stéréotypes mobilisés y dépendent du tour plus personnel et plus affirmé qu'y prennent des échanges, caractérisés par une écoute mutuelle plus impliquante.

Karl et Louise nous ont invitée à les rejoindre à leur domicile un dimanche afin de partager un moment convivial en leur compagnie, avec deux autres couples d'amis pieds-noirs. Karl, un informateur particulièrement coopératif, nous avait consulté afin de savoir de quelle manière nous souhaitions être présentée aux autres invités et si nous avions des requêtes particulières à leur soumettre. Surprise par sa demande et prise au dépourvu, nous lui avions suggéré de proposer à ses amis d'apporter l'un de leurs objets préférés leur rappelant l'Algérie.

Le jour dit, nous retrouvons donc le couple et leurs amis. Avant leur retraite, Karl et Louise étaient instituteurs, Roger exerçait la profession de pharmacien, Monique, son épouse, était femme au foyer, Henri cadre chez EDF ${ }^{15}$.

Après les salutations et présentations d'usage, nous nous installons tous dans le salon. Nous employons le verbe " travailler » à propos de notre recherche, ce qui suscite une plaisanterie de Roger sur le fait que le dimanche est un jour chômé. Celle-ci amène aussitôt une anecdote mobilisant des stéréotypes associés au rapport au travail des Méditerranéens. Karl invite alors ses amis à recentrer le cours de la conversation sur les raisons de notre présence et demande à ce que chacun présente l'objet qu'il a apporté. Cela déclenche chez Roger une énumération des quelques objets conservés depuis l'Algérie. Il souligne ensuite le peu d'intérêt de ceux-ci du point de vue de leur degré d'exotisme : "c'était comme ici, nous avions tout comme ici. Comme tout

15 Nous ignorons la profession de Micheline (la conjointe d'Henri), unique Métropolitaine du groupe. 
Français à l'étranger, quand nous voulions acheter de la vaisselle, on achetait du Limoges, comme nous l'aurions fait en Métropole». Il évoque «un grand plat en bois pour rouler le couscous »; son épouse ajoute que cet objet « n'a pas fait le voyage vers la Métropole » suggérant ainsi que sa valeur économique ne valait pas les efforts qu'il aurait fallu fournir pour le rapporter. Elle nous fait part d'une pensée qui lui a traversé l'esprit quelques heures auparavant, alors qu'elle se préparait pour cette sortie : seuls un plateau «très joli », un vase « tous les deux [vase et plateau] marocains » et des tapis ont finalement été conservés par son mari et elle. Monique pense probablement à cet instant précis que ce qu'elle présente de son histoire reflète une vérité objective ; nous constaterons toutefois par la suite, lors d'un entretien postérieur mené auprès du couple, que davantage d'objets ont pu être rapportés (notamment une imposante bibliothèque, des meubles, etc.), tandis que d'autres ont été acquis après le départ, parce qu'ils évoquaient l'Algérie.

Monique explique donc qu'elle ne pouvait apporter avec elle son plateau, bien trop encombrant, et qu'elle ne s'imaginait pas rendre visite à ses amis avec un tapis. Elle raconte l'histoire de ce dernier, les conditions de son achat, détaille ensuite sa couleur ainsi que les différents motifs dont il est orné. Les hommes entament un échange sur les manières de tisser propres à la région d'origine du tapis (Tlemcen), tandis que les femmes, assises côte à côte, discutent de l'entretien des textiles artisanaux. La conversation masculine incite alors Roger à décrire le départ de ses parents. Il n'adopte aucunement un ton dramatique, cherchant plutôt à relater des faits sur lesquels ses amis trouveront à leur tour des prises narratives, susceptibles de féconder de nouveaux récits :

Les parents sont restés quelques années en plus, alors ils n'avaient gardé que le strict nécessaire et avaient eu le temps d'envoyer leurs meubles qui étaient déposés dans un garage loué. Ils avaient fait des cadres, ont rapporté ce qu'ils voulaient [...]. Ses parents à elle [Monique] sont partis plus tôt et là, ils ont rien pu prendre. Vous pensez bien qu'ils ont pas pensé à prendre un vase! C'est pareil pour chacun, hein?

Tous acquiescent. Les femmes conversent de choses identiques de leur côté. Monique parle de sa mère ; Henri qui devait prêter une oreille attentive aux propos des femmes demande des nouvelles de celle-ci. Elle lui répond et s'amuse du fait que sa mère joue toujours à la belote. S'ensuit un échange entre tous les membres sur les manières les plus adaptées de tricher à ce jeu de cartes. Ils se moquent alors d'un ami commun qui pratique le bridge avec eux : "Gérard, il est "con", comme on dit chez nous ! » lance Roger au cours de cette conversation. Nous supposons alors que ces amis ont fait connaissance par le biais d'une association. Nous intervenons pour la première fois ${ }^{16}$ et demandons s'ils se sont rencontrés lors de parties de bridge comme nous avons cru le comprendre. En guise de réponse, Roger rapporte une anecdote concernant une partie que Karl aurait brillamment remportée (mais dont l'intéressé ne se souvient pas). Sans prêter davantage d'attention à notre précédente question, les hommes reprennent le cours de leurs échanges. Roger raconte maintenant de quelle manière il a acheté sa

16 Après vingt-cinq minutes, au cours desquelles nous nous sommes efforcée de demeurer en retrait afin de laisser les amis échanger le plus spontanément possible. 
maison à un employé de banque pied-noir. Henri se souvient que la première fois qu'il s'est rendu chez Roger, il s'est blessé à la tête en descendant dans la cave. Ensemble, ils situent la maison de Roger et Monique pour Karl qui ne voit pas l'endroit précis où elle se trouve. Ils la localisent près d'une boulangerie. Henri évoque alors un ami commun, un personnage truculent, nommé « Lulu la boulange ».

Hommes et femmes discutent davantage entre eux. Nous remarquons que les temps de parole mixtes demeurent très inégalement répartis entre les individus. Roger et son épouse monopolisent l'essentiel de l'espace sonore ; les interventions du couple influent fortement en outre sur le déroulement des échanges. Henri se manifeste, quant à lui, de manière plus sporadique, en formulant quelques plaisanteries. Karl, beaucoup plus posé intervient de temps à autre pour trancher, pour apaiser ; les échanges reprenant plus calmement après chacune de ses modérations. Les deux autres femmes demeurent presque muettes.

Il nous faudra reposer notre question sur la manière dont les six amis se sont rencontrés. Contrairement à ce que nous avions initialement saisi en les écoutant parler, ce sont les femmes qui ont d'abord fait connaissance au sein d'une association locale accueillant les nouveaux arrivants dans la ville. Elles y participaient toutes trois à la section de bridge. Elles se sont rapprochées lorsqu'elles ont compris qu'elles étaient pieds-noirs (à l'exception de Micheline dont seul l'époux est originaire d'Algérie) sans pour autant que, dans ce contexte associatif précis, ce trait commun soit érigé en revendication identitaire. Karl précise :

C'est pas le fait d'être Pied-Noir qui nous a réunis. Nous n'avons pas cherché des Pieds-Noirs mais, quand on en a trouvé, on est allé vers eux. On fait tous ici partie d'une association, mais nous n'y allons jamais.

- Vous vous y êtes rencontrés?

- Non, non. Nous n'y allons jamais. Je ne savais pas jusqu'à il y a peu de temps qu'Henri en faisait partie! Nous n'avons pas cet esprit communautaire, je crois. Les Pieds-Noirs dans le Sud de la France, c'est différent, ils ont cherché à rester entre eux. Ils sont restés parce qu'il y a le soleil, mais aussi parce qu'ils ne veulent rencontrer que des Pieds-Noirs.

Roger acquiesce :

C'est vrai qu'on va aux "Anciens" de notre lycée et on voit les mentalités différentes selon que les gens sont aujourd'hui dans le Sud ou dans le Nord.

Tous confirment ces propos. Nous les interrogeons ensuite sur ce qui fait la cohésion de leur groupe. Karl suggère que le bridge a initialement beaucoup compté, ce à quoi Roger et Monique ajoutent que ce loisir s'est toujours révélé un facteur efficace d'intégration, quels que soient les lieux dans lesquels ils ont pu résider.

Nous les questionnons maintenant sur leurs origines géographiques. Roger enchaîne :

Vous avez la chance de voir présents ici le meilleur et le pire : tout est représenté : Constantine, Alger et les Oraniens !! [rires].

Henri poursuit :

Les Pieds-Noirs, aussi, le plus important, c'est le téléphone arabe, même les non Pieds-Noirs, il nous disent : " tu sais, machin il est de chez vous »! On est 
attiré, faut bien le dire... Il y a les affinités qu'on a ensemble. En Algérie, on se serait sans doute fait la gueule parce qu'on venait de villes différentes, mais là on s'adore parce qu'on est à l'étranger.

Les liens ainsi noués par le biais de leur loisir favori leur ont permis de rencontrer des personnes appartenant à des milieux sociaux dotés de volumes de capitaux équivalents, produisant des attaches globalement fondées sur une homophilie. Par ailleurs, si Karl précise « ne pas avoir un esprit communautaire », cela signifie aussi, en creux, que leur réseau relationnel est déjà suffisamment étendu et varié, leur capital social suffisamment élevé, pour ne pas nécessiter un repli dans un entre-soi pied-noir.

Ces propos rapportés ne représentent pas plus d'une trentaine de minutes sur les quatre heures et demie passées ensemble. La sélection opérée dans notre description vise d'une part à rendre compte de la manière dont s'enchaînent les échanges. Elle met d'abord en lumière la nécessité pour les partenaires de maintenir un flot de parole continu : la plupart du temps la remarque de l'un permet avant tout à un autre de rebondir et de reconduire la conversation. D'autre part, ces scènes témoignent du rôle des relations amicales dans le mouvement de formation et d'expression contextualisées des mémoires individuelles.

Le fait que les amis soient Pieds-Noirs n'apparaît pas de manière significative dans le contenu de ces premiers échanges : les conditions du départ d'Algérie n'auraient vraisemblablement pas été explicitées avec autant de précisions si nous n'avions pas été présente. L'unique référence spontanée à l'Algérie est constituée par la remarque amusée que nous avons précitée : « il est "con", comme on dit chez nous », que Roger prononce sur un ton qui laisse entendre à tous les convives, qu'au-delà de la boutade, le « chez nous » renvoie à un « chez soi » commun, qui consolide le sentiment d'une appartenance collective partagée.

Nous ne pouvons pas évaluer précisément l'influence de notre présence sur ce qui s'est produit au cours de cet après-midi d'observation. Toutefois, à de nombreuses reprises, il nous a semblé que les convives se comportaient d'une manière spontanée, proche de celle dont ils auraient pu le faire en notre absence.

A l'instar de tous les autres thèmes abordés, ceux relatifs à l'Algérie interviennent souvent au hasard des échanges, parce qu'une remarque de l'un des amis fait, par exemple, « remonter » une anecdote à la mémoire d'un autre convive. Tous semblent parfaitement saisir l'ensemble des significations - y compris celles qui n'ont fait l'objet d'aucune explicitation - sans que le locuteur n'ait besoin de situer spatialement ou temporellement les événements relatés. Ainsi, d'une manière identique à ce qui se dit lorsqu'il est question de leur ville de résidence dans les années quatre-vingt et de leur ami «Lulu la Boulange », chacun dispose des éléments implicites indispensables à la compréhension des échanges. Quantité de significations et d'opérateurs de cadrage sont donc supposés partagés, procédant d'un «stock commun » de « connaissances disponibles [qui] fonctionnent comme schèmes de référence » (Schütz 1994 : 12), sur lequel s'appuie le sentiment de l'entente amicale.

En outre, le fond d'évidences sur lequel prennent racine les conversations décrites, autorise d'étranges jonctions temporelles. Dans les récits, passé et présent, s'entremêlent sans solution de continuité, dans une synthèse narrative comprimant les temporalités et laissant ouvert le champ des interprétations possibles. Chacun situe immédiatement et sans aucun effort la date approximative du départ d'Algérie du locuteur, l'époque à 
laquelle les hommes fréquentaient leur ami boulanger, etc. La possession d'un stock de connaissances communes (qui favorise le sentiment du partage) autorise des échanges qui contribuent à nourrir les impressions d'unité de l'expérience et le sentiment de continuité de soi, liant en un seul fil temporel l'Algérie, la Métropole, le passé de l'enfance, les années 1980, comme la veille.

On constate de surcroît que les stéréotypes, appropriés antérieurement, trouvent à s'exprimer dans ce contexte de conversation banale. Leur caractère opératoire renforce de la sorte le sentiment de l'appartenance culturelle commune. Certaines typifications apparaissent de manière explicite ; c'est ici le cas de l'opposition Oran-AlgerConstantine, par exemple, qui, mobilisée ici et maintenant, renvoie à des antagonismes très prégnants aux temps de l'Algérie française. D'autres, plus fines, nourrissent les jeux complexes de délimitation du groupe. Ainsi, au détour d'une remarque qui se veut humoristique (les "Arabes tirer[erai]ent au flanc »), nous saisissons que nos interlocuteurs ne se considèrent pas comme des "Arabes " puisque ceux-ci sont porteurs d'un stigmate volontiers moqué. Ce type de catégorisation, ce " racisme tout simple [...], le typique racisme français » (Cardinal $1994: 35)$, consacré par les lois de la République en Algérie française, repose sur l'efficace de visions du monde profondément intériorisées dans le creuset colonial. Nous observons ainsi, chez ces amis, mais aussi dans de nombreux entretiens, qu'aujourd'hui encore, le sentiment de supériorité s'est rarement affaibli et joue bien souvent à plein lorsqu'il s'agit d'établir la frontière entre « Eux » (en l'occurrence, les Algériens) et « Nous » (les Français).

Pour autant, cette frontière apparaît difficile à fixer, voire même à cerner ; les interactions amicales rapportées donnent la mesure de la labilité pratique de l'identité, c'est-à-dire, telle qu'elle se produit en situation. Nous l'avons dit, ces amis ne se voient pas comme des «Arabes ». Les extraits rapportés montrent qu'ils peuvent temporairement se définir par leur ville d'origine, puis finalement comme des « Français ayant vécu à l'étranger » (ce qu'administrativement ils n'étaient pas), ou bien comme des « Français aux pratiques identiques à celles des Métropolitains » (qui, « comme tout Français » achèteraient de la porcelaine de Limoges), ou comme des « Pieds-Noirs », ou encore, plus exactement, comme des Pieds-Noirs différents de ceux du Sud de la France, etc. On constate que pour un même individu, le positionnement identitaire peut varier en fonction de la dynamique propre à l'interaction, comme des caractéristiques des personnes en co-présence. Par exemple, nos interlocuteurs ne se sentiront jamais autant « Français » que face à des «Algériens », jamais autant « Français d'Algérie » ou « Méditerranéen » que face à des « Français de France », jamais autant « Constantinois » que face à un "Oranien » ou encore jamais autant "Pieds-noirs refusant l'esprit communautaire » que face à un " Pied-Noir» membre d'une association, etc. Ce même fil pourrait être ainsi déroulé longuement. Les appartenances (et les conséquences qu'elles emportent) peuvent alors tantôt être adoptées pour leur propriétés enviables, tantôt apparaître comme des repoussoirs, sans pourtant que l'individu n'ait jamais l'impression de se déjuger.

On comprend donc que la mobilisation banale de stéréotypes partagés se réalise de manière souple et contextualisée. Les actualisations de ces derniers dépendent des cadres de l'interaction. Enfin, le degré d'intimité ou l'occasion de la rencontre pèsent fortement sur le cours des échanges banals. 
A la différence de ce que nous avons observé au sein des associations, au cours des interactions amicales, les interloculteurs se préoccupent davantage de prendre en compte la spécificité de l'autre dans la communication. D'une manière générale, les discussions se font moins décousues, plus « fluides ». Après avoir assisté à ces différentes scènes, il nous a donc semblé que le mode de relation qui a cours dans le contexte associatif s'inscrit davantage dans un ordre formel : si les interactions y relèvent d'un mode privé, toutefois le fait de parler de l'Algérie pourrait suffire à l'émergence d'une sociabilité entre les personnes présentes. Participer à ce type de réunion à vocation communautaire permet d'une part d'évoquer librement l'Algérie, thème fédérateur qui devient le plus petit dénominateur commun liant les interactants, y compris si ces derniers ne se connaissent que peu, voire pas. D'autre part, les réunions festives associatives constituent des occasions de réactiver des manières d'être stéréotypiques, dont l'usage de termes pataouètes, l'accent parfois forcé ou la présence de nourriture fortement connotées identitairement suffiraient a minima à témoigner. De la sorte, les signes de la communication à tonalité mnésique et identitaire priment fréquemment la construction d'un échange discursif mieux circonscrit. Or, entre Karl, Louise et leurs amis, les évocations directes de la vie algérienne s'imposent moins souvent dans le cours de la conversation ${ }^{17}$. La configuration de cette rencontre amicale (réunissant trois couples) n'est pas toutefois apparue propice à l'expression de sentiments personnels. Conformément à ce qui a été observé concernant les propos tenus entre Pieds-Noirs dans les associations, le tour léger et hédonique caractérisant la plupart des conversations nécessite parfois d'oblitérer ou d'atténuer les aspects les plus douloureux de certains pans de son histoire. M. Pollak, bien que dans un tout autre contexte, insiste ainsi sur le fait que " le silence sur soi -différent de l'oubli -peut même être une condition nécessaire de la communication » (1993 : 38). En ce sens, si ces Pieds-Noirs éprouvent du plaisir à discuter entre eux, l'essentiel de ce qui les unit réside surtout dans le fond commun qui compose une compréhension mutuelle. Le lien affectif passe par l'entente actuelle, la convivialité et la croyance dans le partage d'un passé commun, parfois plus imaginé que véritablement connu.

La plupart du temps, en particulier lorsque les relations ne reposent pas sur des liens de proximité suffisamment forts, la mémoire qui s'exprime apparaît comme lissée, policée et sans anicroche. Une interlocutrice, par exemple, nous a confié avoir été invitée à plusieurs reprises à participer à des réunions associatives. Bien que jusque là, elle se soit toujours montrée enthousiaste à l'idée de rencontrer d'autres Pieds-Noirs, elle explique que ses positions politiques (elle est communiste, favorable à l'Indépendance de l'Algérie) lui ont valu quelques remarques acerbes de la part d'autres participants. Désormais, elle adopte une position de refus très vif, les Pieds-Noirs participant à la vie associative allant jusqu'à lui servir de repoussoirs identitaires.

Ainsi, seuls les aspects qui paraissent les plus « acceptables » selon les individus en co-présence sont évoqués. Toutefois, au cours de ces conversations sont répétés (ou sous-entendus) des éléments stéréotypiques qui nourrissent les mémoires de chacun sur le mode du « reminiscing » dont $\mathrm{P}$. Ricœur explique qu'il « consiste à faire revivre le passé en l'évoquant à plusieurs, l'un aidant l'autre à faire mémoire d'événements ou

17 Elles occupent approximativement trois-quarts d'heure sur les quatre heures et demie - si l'on exclut les propos suscités par nos questions. 
de savoirs partagés [...]. La forme canonique du reminiscing est la conversation sous le régime de l'oralité : « Dis, tu te souviens de..., quand... tu... nous... ? »» (2000 : 46-47).

\section{Conclusion}

La spécificité de cette recherche consistait à mettre au jour une partie du travail permanent d'appropriation et d'actualisation de la mémoire ; un processus à la fois banal et quotidien, réalisé dans l'immédiateté et le présent de différents contextes micro-sociaux privés. En nous centrant sur deux formes ordinaires de sociabilité, notre démarche visait ainsi à prendre au sérieux ce que nous nommons une « petite production mnésique ». Bouillon de culture se dévoilant dans les plis du quotidien, cette dernière prend forme, en deçà des mémoires officialisées, dans les pratiques ordinaires et les environnements matériels (e.g. objets domestiques banals) et humains (e.g. bavardages quotidiens) les plus immédiats des enquêtés.

Il ne s'agit pas pour autant de revendiquer pour cette mémoire une pleine indépendance à l'égard des autres niveaux de mise en forme mnésique (produits dans les champs politique, scientifique, artistique, par exemple), qui, comme le montrent les approches classiques, lui fournissent cadres et contenus (Halbwachs 1994 ; Candau 1998 ; Lavabre 1994). Nous avons pu constater, en effet, qu'aux paliers du social examinés dans ce texte, les cadres collectifs se donnent de manière immanente, dans la concrétude et l'immédiateté de l'expérience quotidienne. Dans les cas étudiés, ils se manifestent notamment à travers la mobilisation de stéréotypes communs, qui ont cours dans des sous-ensembles du groupe des Pieds-Noirs. Leur expression est favorisée par l'instauration temporaire de certaines conditions interactionnelles. Les relations d'interconnaissance favorisent, en effet, une connivence, qui, certes, contribue au sentiment de partager une identité commune, mais rend aussi possible la mobilisation et la circulation des catégorisations évoquées. Nous avons alors pu montrer qu'au cours d'interactions banales, des éléments, sinon vrais, du moins plausibles, se maintiennent, sont réévalués ou introduits dans la mémoire individuelle. Ceci signifie que l'actualisation des cadres et contenus mnésiques, à la fois reconduits et amendés au gré de ces échanges, obéit aux dynamiques propres aux interactions ordinaires, qui à ce titre, apparaissent comme des sources relativement autonomes de la « petite production mnésique ».

\section{Références citées}

Anderson, Benedict, 1983. Imagined Communities. London : Verso.

Bastide, Roger, 1970. « Mémoire collective et sociologie du bricolage », L'année sociologique, 21 : 65-108.

-, 1995 [1960] . Les religions africaines au Brésil. Contribution à une sociologie des interpénétrations de civilisation. Paris : PUF.

Berger, Peter et Thomas Luckmann, 1996 [1966]. La construction sociale de la réalité. Paris : Armand Colin.

Bidart, Claire, 2006. "Les formes de l'amitié », in Xavier Molénat (coord.), L'individu contemporain, Regards sociologiques, 214-225. Paris : Sciences Humaines Editions.

Buono, Clarisse, 2004. Pieds-Noirs de père en fils. Paris : Balland. 
Candau, Joël, 1998. Mémoire et identité. Paris : PUF.

-, 1999. Epistémè du partage. Nice : Mémoire d'habilitation à diriger des recherches.

Cardinal, Marie, 1994. Les Pieds-Noirs. Paris : Furstemberg

Comtat, Emmanuelle, 2009. Les Pieds-Noirs et la politique : quarante ans après le retour. Paris : Presses de Sciences Po.

Connerton, Paul, 1989. How Societies Remember. Cambridge : Cambridge University Press.

Halbwachs, Maurice, 1994 [1925]. Les cadres sociaux de la mémoire. Paris : Albin Michel.

-, 1997 [1950]. La mémoire collective. Paris : Albin Michel.

Herzfeld, Michael, 1997. Cultural Intimacy : Social Poetics in the Nation-State. New York : Routledge.

Hobsbawm, Eric, 1983. The Invention of Tradition. Cambridge : Cambridge University Press.

Javeau, Claude, 1996. « Parler pour ne rien dire. « Ca va ? Ca va !»», Ethnologie française XXVI : 255-263.

Jenkins, Richard, 2008. Rethinking Ethnicity. Los Angeles : Sage publications.

Lavabre, Marie-Claire, 1991. « Du poids et du choix du passé. Lecture critique du « Syndrome de Vichy », in Denis Peschanski, Henri Rousso, Michael Pollak (dir.), Histoire politique et sciences sociales. Paris : CNRS, Cahiers de l'Institut du Temps Présent : 265-278.

-, 1994. Le fil rouge. Sociologie de la mémoire communiste. Paris : Presses de la fondation nationale des sciences politiques.

Piette, Albert, 1996. Ethnographie de l'action : l'observation des détails. Paris : Métailié.

Pollak, Mickael, 1993. Une identité blessée. Etudes de sociologie et d'histoire. Paris : Métailié.

Ricoeur, Paul, 2000. La mémoire, l'histoire, l'oubli. Paris : Seuil.

Savarèse, Eric, 2002. L'invention des Pieds-Noirs. Paris : Séguier.

Schütz, Alfred, 1994 [1971]. Le chercheur et le quotidien. Phénoménologie des sciences sociales. Paris : Méridiens Klincksieck.

Stora, Benjamin (dir.), 1993. Histoire de la Guerre d'Algérie (1954-1962). Paris : La Découverte.

Tabois, Stéphanie, 2005. "Les usages biographiques des écrits pied-noir », in Lila Ibrahim-Lamrous, Catherine Milkovitch-Rioux C. (dir.), Regards croisés sur la guerre d'Algérie, pp. 209-220. ClermontFerrand : Presses de l’Université Blaise Pascal. 